\title{
Cancer Trials Support Unit
}

National Cancer Institute

\section{Source}

National Cancer Institute. Cancer Trials Support Unit. NCI Thesaurus. Code C20090.

Streamlines and centralizes administrative, financial, and data collection tasks. The submission and development of ideas for protocols are made easier through electronic forms, templates, and databases. 